Digital Press Social Sciences and Humanities

Analyse des discours des presses régionales sur les attentats à Paris et à Nice

Rosidin Ali Syabana

Proceeding of Conférence internationale sur le français 2018

Joesana Tjahjani, Merry Andriani, Sajarwa, Wening Udasmoro (eds) 


\title{
Analyse des discours des presses régionales sur les attentats à Paris et à Nice
}

\author{
Rosidin Ali Syabana \\ Universitas Gadjah Mada, Yogyakarta, Indonesia \\ e-mail : rosidin.ali.s@mail.ugm.ac.id
}

\section{Résumé}

Après l'ère de la colonisation, la France commence à recevoir plusieurs d'immigrant venant de quelques pays Afriques et Moyen-Orients. Il immigre en France pour plusieurs raisons tels que, le travail, l'éducation, la meilleure qualité de la vie, etc. Comme il est minorité, il souffre quelques discriminations comme le juif, le noir, l'homosexuel, et les autres communautés mineures. Cette tension attribuée au musulman dont le nombre a augmenté après la révolution irakienne, l'affaire de Salman Rushdie, et le phénomène 9/11 aux États-Unis. Ces événements montrent que le phénomène de l'islamophobie évolue à la société française. Le plus choc tragédie a passé en 2015 et 2016 quand le terroriste massacre plus de 200 personnes à Paris et à Nice. Nous supposons que le media aurait dû donner le calme à la société par rapport à cette tragédie. Mais, en vérité il diffuse également le discours de l'islamophobie en utilisant quelques mots négatifs, comme djihadistes, attribués à musulman. En utilisant l'analyse du cadrage du discours de Robert M.Entman, notre recherche parle de discours des cinq médias régionaux français qui reportent le phénomène de l'attaque terroriste à Paris et à Nice. Nous supposons que la majorité de médias français utilise le mot négatif pour diminuer l'image de l'islam radical qui se grandisse au pays Moyen-Orients. De plus, ils montrent que l'islamisme en France n'est pas la même chose avec celui du terroriste.

\section{Mots-clés}

Islam, France, terrorisme, l'analyse du discours, presse

\begin{abstract}
After the era of colonization, France began to receive immigrants from some African and Middle East countries. They immigrated in France for several reasons such as work, education, quality of life, etc. As they are minority, they suffer from discrimination as experienced also Jews, Black people, Gay, and other minority communities. This tension attributed to the Muslim has increased after the Iraqi revolution, the case of Salman Rushdie, and the 9/11 phenomenon in the United States. These events show that the phenomenon of Islamophobia is changing French society. The most shocking tragedy happened in 2015 and 2016 when terrorists slaughtered more than 200 people in Paris and Nice. We assume that the media should give calm to society compared to the tragedy. But in truth it also diffuses the discourse speech of Islamophobia by using negative words, as jihadists, attributed to Muslim. Using the framing discourse analysis of Robert M. Entman, our research speaks of discourse of three French media reporting the terrorist attack in Paris and Nice. We assume that the majority of French Media uses negative words to diminish the image of radical Islam who will grow up in the Middle East countries. In addition, they show that Islamism in France is not the same with that of the terrorist
\end{abstract}

\section{Keywords}

Islam, France, terrorisme, discourse analysis, press 


\section{Introduction}

Le grand massacre qui a eu lieu en 2015 et 2016 à Paris et à Nice a tué plus de 200 français. Le président à ce moment-là, François Hollande, avec les politiciens français, la communauté musulmane française, et le chef de plusieurs pays étrangers en ont exprimé leur condamnation et bouleversement, en même temps le soutien aux victimes, dans le média. Selon le rapport, ces attaques viennent de pays Moyen-Orient, particulièrement par l'Etat Islamique Irakienne (France Info, 2016). Si nous tirons l'histoire d'immigration en France, La France a l'histoire proche du terrorisme depuis longtemps. Nous pouvons trouver l'immigration post-colonie étant l'un de sources du terrorisme se passant après l'âge de colonisation en France où plein de personnes maghrébines y ont été allées avec son but différent (la famille, le travail, l'éducation, etc.) (Buijs \& Rath, 2006).

Cette attaque a été associée au groupe terroriste islamique se déroulant au Moyen-Orient. Elle met la relation entre le musulman en France et le français dans la plus grave condition possible. Elle positionne le musulman dans la position marginal et négatif face au public parce qu'elle construit le stéréotype et le préjudice qui le méprise en tant que le terroriste et la personne barbare (Strabac \& Listhaug, 2008). Le cas de Charlie Hebdo, l'attaque à Paris et Lyon en 1995 et 1996, et l'attaque terroriste à Paris et à Nice en 2015 et 2016 sont quelques exemples de cas qui met le musulman dans la position marginal. Le sociologiste Farhad Kosrokhavar dit que la France est la prison primaire à la religion d'Islam à cause de sa population étant nombreux dans la prison à la banlieue de Paris (Bleich, 2009). L'islam en France est aussi considéré en tant que la première cause d'inégalité sociale et économique à cause de son culture et ethnicité (Boukhars, 2009).

Dans la scène international, plein d'acteurs considère que l'islam est une religion barbarie et radicale. Le conflit civil dans le pays Moyen-Orient, conjointement aux controverses de roman de Salman Rushdie intitulé Les Versets Sataniques, la révolution iranienne, et la tragédie 9/11 aggrave l'image de l'islam au public internationale. Depuis ce moment, le sentiment du français au musulman commence à s'épanouir parce que ceux-là ont le lien avec le musulman. Par conséquence, la société occidentale la voit comme une acte barbarie et inhumaine (Said, 1997). De plus, la laïcité semble d'alourdir de la communauté musulmane en France à cause de la différente interprétation de chaque culture (Abdelaziz, 2018; Huntington, 2000 ; Statham, 2016).

Tous les phénomènes d'abord ont la même structure que l'Islam est la religion ayant le lien fort avec la violence pour résoudre les problèmes. Cela pourrait invoque l'islamophobie, la haine à l'islam et le musulman, en Europe, notamment en France de sorte que les musulmans souvent souffrent la discrimination dans la rue à cause de son symbole religieuse comme la viole (Helbling, 2014). Toutes les attaques sont attribuées au musulman, même s'il ne soutient jamais la violence en ressoudant le problème. Ce stéréotype se déroule dans la vie quotidienne de musulman en France, notamment l'attaque à Paris et à Nice. La haine à l'Islam pourrait être plus grave parce que cette tragédie a menacé la sécurité nationale et la société. Pourtant, ce qui diffuse ce discours est la presse qui utilise quelques stratégies discursives pour donner l'information à société se consistant de mots, phrase, et lexique ayant le discours à diffuser.

La presse construit le bon sens en utilisant cette stratégie discursive donc la société croit ce que le média lui informe. Il diffuse constamment son discours pour que la société suive la pensée de média (Perse, 2001 ; Tuchman, 2000). Même si la société a son filtre via l'éducation formelle dans la méthode de choisir et d'accéder le journal, à cause de l'internet et la vite diffusion des informations aujourd'hui, la société est encore piégée par l'hégémonie de média. C'est parce que le média utilise aussi la méthode de l'institutionnalisation et la légitimation pour diffuser les informations (Berger \& Luckmann, 1966).

L'autre stratégie que le média utilise est l'assortiment au responsable ou à la ressource plus extrême, par exemple le chef de l'équipe terroriste islamique, en réclamant qu'il est la vraie représentation de l'islam (Poole, 2002). Said (1997) explique comment le média occidental construit le discours clair sur l'identité de l'islam dans le point de vue de l'Occident. Par exemple, quand la révolution iranienne en 1979 se passait, beaucoup d'employées de l'ambassade américaine y a été kidnappé. Ce phénomène construit un discours parmi la société européen qu'Iran, comme la représentation de pays musulman, est un pays barbarie, irrationnel, autoritaire, et primitive. Dans ce cas, l'islam est l'autre, et ce que le média suscite dans le public est l'image négative donc le français l'associe avec le concept violent, menaçant, et opposé l'Occident (Poole, 2002; Said, 1997). 


\section{Méthode}

Cet article voulait parler de comment les presses régionales français répondent à la question du terrorisme, notamment se passant en 2015 et en 2016 à Paris et à Nice. Il se divise dans deux discussions. Le premier chapitre discute les lexiques utilisés par cinq médias en regardant son contexte. Ce sont La Montagne, Presse Océan, Actu Toulouse, Le Point, et Ouest France. Ces presses sont choisies grâce à sa qualité de reportage et sa popularité parmi le français. Les cinq presses analysées montrent la quantité différente en utilisant le mot spécifique. Nous analysons le mot étant plus fréquent que les autres basé sur la calculassions dans Lexico. Il montre que ces mots à le contexte orienté au la communauté spécifique dans la tragédie. L'application révèle aussi le lexique en valeur positif pour donner le sentiment sécurisé à la société. Nous analysons ce mot avec son contexte pour essayer de comprendre le discours qu'un article veut diffuser dans la société. Pour conclure ce chapitre, on peut tirer la pensée comment le rôle de presse dans le temps difficile et urgent en France, notamment le terrorisme.

Le deuxième chapitre discute le discours dans un texte. Il est différent de celui de la première partie parce que chaque texte est analysé pour trouver son discours. En utilisant la méthode d'Entman (1997), cette partie essaye de montre le choix de mots et l'assortiment du thème qu'un discours veut diffuser (Perse, 2001 ; Entman, 1991). Entman pense qu'un cadrage a quatre étapes de mesure ; le problème, la cause, le jugement moral, et la recommandation du traitement. Pourtant, celle étant le plus important est le jugement moral parce qu'il utilise de l'adjective étant plutôt subjective (Entman, 2007). Dans dix articles de chaque presse analysée, nous pouvons trouver comment cette presse juge la tragédie en regardant le contexte plus grand que la première discussion. En fin, ce chapitre peut dire comment l'idéologie de chaque presse face à le terrorisme en France.

\section{Résultat et discussion}

\subsection{La répond lexique aux attentats à Paris et à Nice par la presse}

Le choix de mots est un aspect important pour construire un discours dans le texte. Il peut former le bon sens parmi la société à cause du processus de l'institutionnalisation (Berger \& Luckmann, 1966). Le journaliste a son propre rôle pour sélectionner ces choix de mots dans son article. C'est facile à trouver le cas quand le journaliste abuse son pouvoir pour qu'il puisse gagne beaucoup de cliques en écrivant le titre fallacieux dans l'internet, même dans l'ère de la technologie vaste, sophistiquée, et efficace, sa quantité se montre rapidement. Tandis que la société a le choix pour filtrer la nouvelle et l'information, elle est encore dans une hégémonie de ce titre fallacieux à cause de la vitesse de sa diffusion.

Les énoncés et le lexique ont le lien proche entre l'un et l'autre. Ils se constituent dans la partie sociocognitive pour générer le discours dans la société. Le texte contient la connaissance pour être diffusée dans la forme physique ou digitale. Ce qui a le pouvoir de choisir le lexique est le journaliste, parce qu'il partage sa compréhension d'un évènement en utilisant le topique spécifique, l'utilisation de la forme passive ou active, le lexique choisi, et les autres à la société (Dijk, 2015). Ce texte se diffuse tous les jours parmi la société où elle le lit dans sa quotidienne. Ce mot, cet énoncé, et cette connaissance se reproduit également par la presse donc il peut gérer la pensée de la société sur un évènement.

Dans le contexte de l'attaque à Paris et à Nice, les presses régionales jouent le grand rôle pour informer la plus vite possible ce qui se passe dans le lieu du crime. Mais, le lexique choisi est intéressant parce que quelques mots s'emploient pour accuser certaines communautés minorités en France. Parmi les cinq presses analysées, celle étant plus vocal en reportant l'attaque est Le Point, le média régional à Paris, et aussi le média qui utilise le plus mots en valeur négatif que les autres médias. C'est justifiable parce qu'il est le média étant plus proche du lieu du crime, donc la trafique de reportage pourrait être grande. En même temps, La Montagne, le média régional à Clermont-Ferrand, est le plus passif média reportant la scène. Nous trouvons que sept lexiques ont le contexte plus intéressant à analyser. Ils sont aussi plus fréquents à son utilisation dans le texte. Ce sont attentat(s), victimes, notre, femme, Daëch, sécurité, et terroriste. 
Tableu 1 Les lexiques choisis dans les rapportages

\begin{tabular}{|l|l|l|l|l|l|l|l|}
\hline \multirow{2}{*}{$\begin{array}{c}\text { Nom de } \\
\text { Presse }\end{array}$} & \multicolumn{7}{|c|}{ Mots utilises dans le reportage } \\
\cline { 2 - 9 } & Attentat(s) & victimes & notre & femme & Daëch & sécurité & terroriste \\
\hline La montagne & 21 & 11 & 10 & 0 & 1 & 7 & 1 \\
\hline Presse océan & 30 & 19 & 15 & 6 & 4 & 7 & 7 \\
\hline $\begin{array}{l}\text { Actu } \\
\text { Toulouse }\end{array}$ & 30 & 10 & 7 & 0 & 2 & 12 & 0 \\
\hline Le point & 60 & 19 & 12 & 26 & 1 & 9 & 11 \\
\hline Ouest France & 28 & 17 & 3 & 20 & 32 & 5 & 15 \\
\hline
\end{tabular}

Chaque presse a sa propre utilisation de mots dans son reportage. Nous pouvons voir dans le tableau 1 que l'utilisation de mots attentat(s), le mot ayant le sens sémantiquement négatif, est différent de chaque presse. Le Point utilise le plus lexique négatif (72 mots calculés) que les autres presses au point que nous pouvons assumer le bouleversement de cette presse en répondant l'attaque à Paris et à Nice. En même temps, La Montagne utilise moins de mot attentat(s) dans son reportage et elle utilise peu de mots en valeur négatif à informer l'évènement à la société. Nous discutons un de ces lexiques ci-dessous.

Le mot victimes est l'un de mots étant intéressant que les presses régionales françaises utilisent dans son reportage sur l'attaque à Paris et à Nice. Elles l'utilisent dans le contexte positif pour faire le rendre hommage aux victimes des attaques au lieu de lancer la condamnation au malfaiteur du crime pour tuer le citoyen. Dans ce cas, la presse a donné le soutien aux familles ayant son membre tué dans la tragédie. Nous pouvons savoir le rôle de la presse pour qu'elle donne le sentiment sécurisé et solidarisé à la société. Cela se représente bien dans les articles de ces presses en reportant l'attaque à Paris et à Nice.

Tableu 2 L'utilisation de mots victimes dans le reportage de l'attaque à Paris et à Nice

\begin{tabular}{|c|c|}
\hline La Presse & L'utilisation de mots victimes dans le reportage \\
\hline Actu Toulouse & $\begin{array}{l}\text { Après Nice, les Toulousains invités à se rendre place du Capitole pour } \\
\text { une minute de silence. Le maire Jean-Luc Moudenc appelle les } \\
\text { Toulousains à se rendre place du Capitole lundi } 18 \text { juillet, pour observer } \\
\text { une minute de silence en hommage aux victimes de l'attentat de Nice } \\
(17-7-2016) \text {. }\end{array}$ \\
\hline La Montagne & $\begin{array}{l}\text { Nous partageons entièrement l'émotion de la population, et en } \\
\text { particulier de tous les proches des victimes de ces attentats(16-11- } \\
\text { 2015). }\end{array}$ \\
\hline Ouest France & $\begin{array}{l}\text { Les terroristes cherchent à nous diviser. Nous diviser serait leur donner } \\
\text { raison. Nous devons dignité et respect à la mémoire des victimes. } \\
\text { Mais cela veut aussi dire que l'on dise la vérité sur les événements (16-7- } \\
2016) .\end{array}$ \\
\hline Le Point & $\begin{array}{l}\text { Au lendemain de l'attaque sur la promenade des Anglais, des centaines } \\
\text { de personnes se sont recueillies à proximité des lieux du drame pour } \\
\text { rendre hommage aux victimes (16-7-2016). }\end{array}$ \\
\hline Presse Océan & $\begin{array}{l}\text { Le CFCM appelle les musulmans de France à prier vendredi « à la } \\
\text { mémoire des victimes » (15-7-2016). }\end{array}$ \\
\hline
\end{tabular}


Dans le tableau ci-dessus, toutes les presses emploient le mot victimes pour le contexte positif ; une minute de silence, le rendu hommage, etc. le discours montré dans le lexique victimes est donc le problème de la sécurité civile. Le discours de la sécurité joue souvent le grand rôle dans le discours du terrorisme parce qu'il menace non seulement la sécurité de citoyen et des infrastructures, mais également la sécurité nationale. Nous parlons de mouvement anonyme qui peut lancer une explosion n'importe où et cela élève la conscience de tous les états. La sécurité ne parle pas seulement de choses physiques, mais également le sentiment phycologique.

La sécurité physique parle de renforcement de la sécurité au temps d'urgent et d'emploi maximale de polices. Elle parle également de politique de prévention pour minimaliser la puissance de menace à l'avenir. Dans le contexte de l'Europe, le musulman est celui qui souffre ce politique à cause de son image négative parmi l'européen, par exemple la surveillance, l'intervention par l'officiel, et le monitoring (Mythen, Walklate, \& Khan, 2009). Ce politique peut aggraver la position des musulmans qu'y habitent à cause de l'augmentation de discrimination et de stéréotype fait par l'état.

Ensuite, nous parlons aussi de la sécurité phycologique de victimes et des familles. La presse dans l'attaque à Paris et à Nice emploi le lexique victimes pour performer ce type de sentiment. Dans le terrorisme, la peur de citoyen est assez grande à cause de son traumatisme du même évènement du passé et c'est le travail des presses de même que des autres sociétés à donner le sentiment sécurisé aux victimes. Nous pouvons en voir comment la presse régionale française travaille pour calmer la situation parmi la société dans tableau 2 en rapportant l'activité positif en tant que le rassemblement pour rendre hommage aux victimes. Le même exemple se montre dans l'attaque terroriste en Indonésie quand l'explosion et la fusillade s'est passé à Sarinah, Jakarta en 2016. Le peuple s'est réuni dans les réseaux sociaux à donner le soutien en utilisant le lexique Kami Tidak Takut (Nous n'avons pas peur). Cette activité s'est diffusée par les presses ayant le même but avec celle des presses françaises qui est pour calmer la situation.

\subsection{L'analyse du discours cadrage pour discuter comment les médias régionaux en France répondent les attentats à Paris et à Nice}

Le peuple utilise le discours dans la vie quotidienne pour construire le bon sens au champ de force différent (Fairclough, 1995). Le personnage politique joue le discours pour convaincre la société sur un problème et le média a le rôle à la diffusion de ce discours. Dans le cas de l'attaque à Paris et à Nice, les presses régionaux montre quelques discours intéressants. Les thèmes des reportages pendant une semaine de publication se consistent de problème de la sécurité, de l'information général des attentats, des pensées des hommes politiques et du citoyen, etc. Le tableau de diffusion de discours se présente cidessous :

Tableu 3 Le cadrage de reportage dans l'attaque à Paris et à Nice

\begin{tabular}{|l|l|l|}
\hline \multicolumn{1}{|c|}{$\begin{array}{c}\text { Nom de } \\
\text { Presse }\end{array}$} & Thèmes dans les publications & \multicolumn{1}{c|}{ Le jugement moral dans le texte } \\
\hline Actu Toulouse & $\begin{array}{l}\text { Le rendu hommage, la } \\
\text { fortement/renforcement de la } \\
\text { sécurité, et les activités après } \\
\text { les attentats. }\end{array}$ & $\begin{array}{l}\text { La peur a également pu inciter les } \\
\text { Toulousains à ne pas participer à un } \\
\text { hommage organisé dans l'espace public. }\end{array}$ \\
\hline La montagne & $\begin{array}{l}\text { Information générale, la } \\
\text { réaction des attentats par les } \\
\text { témoignages et l'expert, et la } \\
\text { responsabilité de citoyen pour } \\
\text { renforcer la sécurité. }\end{array}$ & $\begin{array}{l}\text { Cet attentat est l'illustration d'un monde } \\
\text { terroriste qui change. Désormais, n'importe } \\
\text { qui, récupéré par la radicalisation, peut } \\
\text { passer à l'acte }\end{array}$ \\
\hline Presse océan & $\begin{array}{l}\text { L'information générale, la } \\
\text { réaction et le témoignage des } \\
\text { citoyens, des politiciens, et de la } \\
\text { communauté musulmane, et le } \\
\text { rendu hommage }\end{array}$ & $\begin{array}{l}\text { L'Association islamique de l'Ouest de la } \\
\text { France (AIOF) a appris « avec un sentiment } \\
\text { empreint de désolation et de tristesse » que } \\
\text { la ville de Nice venait d'être « touchée par } \\
\text { un acte criminel lâche et abject ». }\end{array}$ \\
\hline Ouest-France & $\begin{array}{l}\text { Les malfaiteurs, la victime, et la } \\
\text { sécurité }\end{array}$ & $\begin{array}{l}\text { l'un des meurtriers déclarait avoir voulu } \\
\text { venger les « musulmans tués par des } \\
\text { soldats britanniques ». Lors de son procès, }\end{array}$ \\
\hline
\end{tabular}




\begin{tabular}{|l|l|l|}
\hline & & $\begin{array}{l}\text { ce père de six enfants a déclaré qu'il était en } \\
\text { «mission » en tant que « soldat d'Allah » et } \\
\text { " en guerre contre la Grande-Bretagne », en } \\
\text { invoquant la loi du talion }\end{array}$ \\
\hline Le point & $\begin{array}{l}\text { La racine de l'attaque, l'effet de } \\
\text { l'attentat, l'identité des } \\
\text { victimes, et les réactions des } \\
\text { politiciens }\end{array}$ & $\begin{array}{l}\text { le président François Hollande avait parlé } \\
\text { d'« une attaque dont le caractère terroriste } \\
\text { ne peut être nié ». "C'est toute la France } \\
\text { qui est sous la menace du terrorisme } \\
\text { islamiste » }\end{array}$ \\
\hline
\end{tabular}

Dans la comparaison ci-dessus, on trouve que chaque presse a la différente stratégie d'écriture le reportage face à l'attentat à Nice et à Paris. Mais ils sont d'agrée dans un grand thème de reportage, c'est la menace du terrorisme et la sécurité. Parmi des articles analysés, beaucoup parlent de la réponse de la France face à cette attaque. Plusieurs articles parlent du terrorisme en général, ils ne mentionnent pas la communauté musulmane. Mais, quelques presses le fait, comme Ouest France et Le point. Ils écrivent quelques articles qui potentiellement montrent la haine aux musulmans en France. Les thèmes de ses publications sont différents avec les autres. Dans le tableau 3, on trouve que Le Point (2015) a publié l'article sur la racine de l'attaque étant à cause de la guerre d'Algérie. En même temps, Ouest France (2015) parle beaucoup de liens entre les malfaiteurs et le terrorisme islamiste.

Ouest-France a diffusé quelques articles intéressants à propos de son thème « les malfaiteurs ». Il y a un article qui parle d'une femme kamikaze. L'article diffusé le 19 novembre 2015 a discuté sur la première fois que l'EI a eu envoyé la femme pour se faire exploser dans le publique. Le texte montre que cette technique est très rare dans le mouvement de terroristes. Même si le texte parle du début de la femme kamikaze, la femme dans le territoire terroriste commence déjà depuis longtemps. Même elle est dans la positionne de pouvoir comme American Weather Underground des États-Unis, Brigate Rosse d'Italie, et Rote Armee Fraktion d'Allemagne (Nacos, 2005).

La presse Rennais parle aussi du lien entre le malfaiteur et l'équipe terroriste. Le reportage publié le 16 juillet 2016 parle de l'histoire de mouvement terroriste en France. Mais ces terroristes ont lien avec l'islamisme qu'on peut voir dans l'utilisation de mots dans ce texte. Par exemple dans la phrase «l'un des meurtriers déclarait avoir voulu venger «les musulmans tués par des soldats britanniques ». Lors de son procès, ce père de six enfants a déclaré qu'il était en "mission » en tant que "soldat d'Allah » et " en guerre contre la Grande-Bretagne », en invoquant la loi du talion (Ouest France, 16-7-2016)», la presse utilise le mot venger les musulmans tués par des soldats britanniques, c'est-à-dire que les musulmans sont vindicatifs parce que il tue son victime à cause de ce qui s'est passé dans le passé. L'utilisation de mot en mission en tant que soldat d'Allah confirme sa justification de tuerie. Ce type de publication pourrait diffuser l'islamophobie en France à cause de négatif image de l'islam en France.

La stratégie discursive dont les autres presses utilisent est apparemment différent avec celle d'OuestFrance ou de Le point. Actu Toulouse, La montagne, et Presse océan a rapporté la même chose, le terrorisme. Mais, peu de ses publications parle de terrorisme conduit par le groupe islamiste. Par exemple, dans le reportage de l'attaque à Nice, Actu Toulouse écrit que «La peur a également pu inciter les Toulousains à ne pas participer à un hommage organisé dans l'espace public. "Ce genre de rassemblement génère de la crainte, on évite les foules car elles ont été d'attaques lors des attentats » remarque Teddy (24 ans) (Actu Toulouse, 19-7-2016) ». Dans cette phrase, elle parle de comment le terrorisme incite la peur parmi la société tandis qu'elle habite dans la région différente avec la scène de crime. Même, elle ne veut pas participer dans l'hommage public à cause de la peur à l'attaque soudain.

Ce qui concerne ces presses est comment le cadrage se construit dans le contexte de terrorisme. Il y a deux grands thèmes dans une question qu'elles posent dans leurs publications : qui est coupable ? Le terroriste ou l'islam ? Quelques presses disent le premier, les autres appellent le dernier. La chose pire dans le dernier est comment ce message diffuse le discours d'islamophobie en France et construit le stéréotype négatif au musulman. À cause de l'action de tuerie par l'équipe terroriste et l'histoire en France avec l'immigrant et le monde d'Islam, ce type d'attaque peut cristallise la construction de pensée que l'Islam est la religion barbare. Même si la publication donne l'espace pour les musulmans à parler dans le publique, comme celle d'Actu Toulouse et de Presse Océan, la publication montrant le discours d'islamophobie peut discriminer la position des musulmans en France 


\section{Conclusion}

Les médias régionaux en France font le grand effort de donner le texte informatif et compréhensif. Ils ont les méthodes et les approches différente à rapporter l'issue sensitive comme l'attaque terroriste. Cela peut se trouver dans les lexiques et les discours qu'ils utilisent dans les reportages. Parmi les lexiques utilisés, ils montrent le discours en valeur plus positif que négatif en tant que la sécurité nationale. Le lexique victime, par exemple, est utilisé pour montre la tristesse et le bouleversement à l'attaque terroriste. Même si l'utilisation de mot attentat(s) est plus fréquent que les autres mots, ce contexte est le plus général. Il s'utilise dans le reportage urgent et l'information générale de la tragédie. Ce mot s'utilise aussi pour répondre quelques questions à propos de l'évènement en tant que les victimes et les témoignes.

Le rôle de presses français dans le temps d'urgent peut aussi voir dans le cadrage de son reportage. Quelques presses montrent le sentiment de tristesse, de sympathie, et de l'unité. Les autres montrent la sécurité et le nationalisme. Mais peu des textes analysés montrent le texte exprimant la haine et la peur au musulman. Ils parlent beaucoup du lien entre les malfaiteurs et l'État Islamique, le résultat de l'immigration, et le temps de poste guerre avec l'Algérie. Même si ce n'est pas compris quel musulman dont le texte parle, parce que quelques presses donnent l'espace pour musulman français à parler et à donner le soutien aux victimes de ces attaques, ce type de texte contribue à la diffusion de l'islamophobie en France. De plus, le phénomène de l'islamophobie met le musulman français dans la position moins avantageux. Dans ce cas, la presse a grand rôle de construire ce discours parce que sa position dans la vie social est le premier acteur qui diffuse l'information dans le publique.

\section{Références}

Abdelaziz, R. (2018). Muslims Are Internalizing Islamophobia, And Negative Media Coverage Is To Blame.

Retrieved from https://www.huffingtonpost.com/entry/muslims-islamophobia-media-

coverage_us_5ae73150e4b04aa23f2598d5

Actu Toulouse. (2016). Hommage attentats. Pourquoi les Toulousains étaient-ils moins nombreux pour Nice ? Retrieved from https://actu.fr/occitanie/toulouse_31555/hommage-attentats-pourquoi-lestoulousains-etaient-ils-moins-nombreux-pour-nice_3713718.html

Berger, P. L., \& Luckmann, T. (1966). The Social Construction of Reality. New York: Penguin.

Bleich, E. (2009). State Responses to 'Muslim' Violence: A Comparison of Six West European Countries.

Journal of Ethnic and Migration Studies - J ETHN MIGR STUD, 35, 361-379.

https://doi.org/10.1080/13691830802704517

Boukhars, A. (2009). Islam, Jihadism, and Depoliticization in France and Germany. International Political Science Review, 30(3), 297-317. https://doi.org/10.1177/0192512109105642

Buijs, F., \& Rath, J. (2006). Muslim in Europe: The State of Research (IMISCOE Working paper). Amsterdam. Retrieved from https://hdl.handle.net/11245/1.271693

Entman, R. M. (1991). Symposium Framing U.S. Coverage of International News: Contrasts in Narratives of the KAL and Iran Air Incidents. Journal of Communication, 41(4), 6-27. https://doi.org/10.1111/j.1460-2466.1991.tb02328.x

Fairclough, N. (1995). Critical Discourse Analysis: The Critical Study of Language. London: Longman. https://doi.org/10.2307/329335

France Info. (2016). Comment le camion a-t-il pu circuler sur la promenade des Anglais pourtant fermée à la circulation? Retrieved from https://www.francetvinfo.fr/faits-divers/terrorisme/attaque-au- 
camion-a-nice/comment-le-camion-a-t-il-pu-circuler-sur-la-promenade-des-anglais-pourtant-fermeea-la-circulation_1548357.html

Helbling, M. (2014). Opposing Muslims and the Muslim Headscarf in Western Europe. European Sociological Review, 30(2), 242-257. https://doi.org/10.1093/esr/jct038

Hilgartner, S., \& Bosk, C. S. (1988). No Title. American Journal of Sociology, 94(null), 53.

Huntington, S. P. (2000). The Clash of Civilizations? In L. Crothers \& C. Lockhart (Eds.), Culture and Politics: A Reader (pp. 99-118). Hampshire: Palgrave Macmillan.

Mythen, G., Walklate, S., \& Khan, F. (2009). I'm a Muslim, But I'm Not a Terrorist': Victimization, Risky Identities and the Performance of Safety. British Journal of Criminology, 49(9), 736-754. https://doi.org/10.1093/bjc/azp032

Nacos, B. L. (2005). The Portrayal of Female Terrorists in the Media: Similar Framing Patterns in the News Coverage of Women in Politics and in Terrorism. Studies in Conflict \& Terrorism, 28(5), 435-451. https://doi.org/10.1080/10576100500180352

Ouest France. (2016). Attentat à Nice. Un véhicule lancé pour tuer, une arme déjà utilisée. Retrieved from https://www.ouest-france.fr/societe/faits-divers/attentat-nice/attentat-nice-un-vehicule-lance-pourtuer-une-arme-deja-utilisee-4369088

Perse, E. M. (2001). Media Effect and Society. London: Taylor \& Francis.

Poole, E. (2002). Reporting Islam: Media Representations of British Muslim. London: I.B. Tauris.

Said, E. (1997). Covering Islam: How The Media and The Experts Determine How We See The Rest of The World. New York: Vintage Books.

Statham, P. (2016). How ordinary people view Muslim group rights in Britain, the Netherlands, France and Germany: significant 'gaps' between majorities and Muslims? Journal of Ethnic and Migration Studies, 42(2), 217-236. https://doi.org/10.1080/1369183X.2015.1082288

Strabac, Z., \& Listhaug, O. (2008). Anti-Muslim prejudice in Europe: A multilevel analysis of survey data from 30 countries. Social Science Research, 37(1), 268-286. https://doi.org/https://doi.org/10.1016/j.ssresearch.2007.02.004

Tuchman, G. (2000). The Symbolic Annihilation of Women by the Mass Media. In L. Crothers \& C. Lockhart (Eds.), Culture and Politics: A Reader (pp. 150-174). Hampshire: Palgrave Macmillan.

van Dijk, T. (2014). Critical Discourse Studies: A Sociocognitive Approach. In R. Wodak \& M. Meyer (Eds.) Methods for Critical Discourse Analysis (pp. 62-85). London: Sage. https://doi.org/10.1075/z.184.79dij 